\title{
Erreurs d'identification des agents biologiques à cote de sécurité élevée du groupe de risque 3 par SM MALDI-TOF au Canada : de novembre 2015 à octobre 2017
}

\author{
Pomerleau-Normandin $D^{1}$, Heisz $\mathrm{M}^{1}$, Su $\mathrm{M}^{1 *}$
}

\section{Résumé}

Contexte : La spectrométrie de masse à désorption-ionisation laser assistée par matrice avec analyseur de temps de vol (SM MALDI-TOF) est une technologie de plus en plus utilisée pour l'identification des microorganismes aux fins diagnostiques. Toutefois, des observations empiriques laissent penser que cette technologie est associée à des erreurs d'identification des agents biologiques à cote de sécurité élevée (ABCSE) du groupe de risque 3 (GR3), ce qui augmente le risque d'exposition pour le personnel en laboratoire.

Objectif : Étudier et caractériser les incidents liés à l'utilisation de la SM MALDI-TOF survenus au Canada du 6 novembre 2015 au 10 octobre 2017.

Méthodologie : Les cas à étudier ont été relevés à partir des rapports d'incidents en laboratoire du système de surveillance national de déclaration des incidents en laboratoire au Canada. Les cas admissibles faisaient directement référence à la technologie SM MALDI-TOF ou à l'un des trois ABCSE du GR3 suivants : espèces de Brucella, Francisella tularensis et Burkholderia pseudomallei. Un questionnaire a été mis au point afin d'établir les facteurs de risque pouvant mener à une exposition. Les déclarants des organisations où des incidents admissibles ont été signalés ont été interrogés au moyen de ce questionnaire. Les données ont été consignées dans une feuille de calcul Excel. Une analyse statistique descriptive a été réalisée par la suite pour évaluer les caractéristiques communes et établir les facteurs de risque possibles.

Résultats : Huit incidents admissibles ont été relevés, et 39 employés de laboratoire au total ont été exposés à des ABCSE du GR3. Dans cinq de ces huit incidents, les déclarants ont indiqué que leur appareil était muni à la fois des bases de données cliniques de référence et de bases de données de recherche de référence. Dans six incidents pour lesquels les déclarants savaient quels types de bases de données avaient été utilisés, seule la base de données cliniques avait été utilisée au moment de l'incident, même si les deux types étaient disponibles dans cinq de ces cas. Dans les huit cas, l'exposition est survenue à l'étape de préparation de l'échantillon, les analyses étant réalisées sur table de travaille à découvert, directement sur le spécimen. De plus, dans les huit cas, les spécimens des patients avaient été fournis sans information sur le risque.

Conclusion : Il s'agit de la première étude d'envergure nationale visant à caractériser la nature et l'étendue des incidents en laboratoire impliquant les ABCSE du GR3 liés à l'utilisation de la SM MALDI-TOF permet d'établir les facteurs de risque et fournit des données de base pouvant éclairer les stratégies d'atténuation du risque.

Citation proposée : Pomerleau-Normandin D, Heisz M, Su M. Erreurs d'identification des agents biologiques à cote de sécurité élevée du groupe de risque 3 par SM MALDI-TOF au Canada : de novembre 2015 à octobre 2017. Relevé des maladies transmissibles au Canada. 2018;44(5):123-9. https://doi.org/10.14745/ ccdr.v44i05a04f

Mots clés : SM MALDI-TOF, erreur diagnostique, microorganismes du groupe de risque 3, ABCSE

\section{Affiliation}

${ }^{1}$ Centre de la biosûreté, Agence de la santé publique du Canada, Ottawa (Ontario)

*Correspondance : ming.su@ canada.ca 


\section{Introduction}

La spectrométrie de masse à désorption-ionisation laser assistée par matrice avec analyseur de temps de vol (SM -MALDI-TOF) est une technologie ayant été qualifiée de « révolution en matière d'identification clinique des microbes " (1). L'identification de microorganisme à partir d'une culture cellulaire peut prendre jusqu'à 18 heures, mais la SM MALDI-TOF permet de réaliser la même tâche en environ 15 minutes (2). En raison de sa simplicité, de sa rapidité et de sa grande capacité de traitement, cette nouvelle technologie est de plus en plus utilisée pour l'identification régulière des microbes, tant dans les laboratoires cliniques que les laboratoires de référence (3). Elle permet de poser un diagnostic précoce tout en réduisant grandement le coût de l'analyse (2).

La SM MALDI-TOF d'un microorganisme donné produit un spectre caractéristique, appelé « empreinte de masse peptidique », qui reflète le profil protéinique unique propre au microorganisme en question (qui peut contenir jusqu'à 2000 protéines) (3). Le logiciel de SM MALDI-TOF compare ensuite cette empreinte de masse peptidique au contenu d'une base de données de référence interne, qui contient les spectres des microorganismes connus. Puisque la composition protéique varie d'une espèce bactérienne à l'autre, la production du spectre permet une identification précise (4).

La principale limite de cette technologie est liée aux bases de données de référence, qui doivent contenir le spectre du microorganisme de l'échantillon pour permettre de I'identifier correctement (5). Si le spectre ne fait pas partie de la base de données, l'appareil ne pourra pas identifier le microorganisme ou identifiera un microorganisme similaire. Une identification non exacte peut être problématique; en effet, des microorganismes de la même famille peuvent appartenir à des groupes de risque différents et, par conséquent, être associés à des protocoles de sécurité en laboratoire distincts.

Les microorganismes sont classés de deux manières : en fonction de leur groupe de risque (pathogénicité, virulence, disponibilité des traitements et risque de propagation; voir le tableau 1) et en fonction de leur appartenance à la catégorie des agents biologiques à cote de sécurité élevée (ABCSE), un sous-ensemble d'agents pathogènes humains qui présentent un risque accru en raison de la possibilité qu'on les utilise comme arme biologique (6). Certains des microorganismes des groupes de risque 3 (GR3) et la plupart des microorganismes du GR4 sont des ABCSE.

Si un spécimen contient un microorganisme du GR3 que l'appareil identifie incorrectement comme un microorganisme du GR2, le spécimen pourrait être manipulé sur table de travail à découvert plutôt que dans une enceinte de sécurité biologique. Par conséquent, en cas d'identification erronée, les techniciens de laboratoire risquent de ne pas avoir la protection appropriée, ce qui pourrait entraîner des incidents d'exposition.

Des études ont indiqué que l'identification erronée des microorganismes par SM MALDI-TOF semble être attribuable à l'incomplétude des bases de données de référence utilisées (2,7-26). Deux types de bases de données de référence sont actuellement utilisées au Canada : les bases de données

\section{Tableau 1 : Définition des groupes de risque et exemples d'agents biologiques}

\begin{tabular}{|l|l|l|}
\hline \multicolumn{1}{|c|}{$\begin{array}{l}\text { Groupe de } \\
\text { risque (GR) }\end{array}$} & \multicolumn{1}{|c|}{ Définition (6) } & \multicolumn{1}{c|}{$\begin{array}{c}\text { Exemples d'agents } \\
\text { biologiques }\end{array}$} \\
\hline RG1 & $\begin{array}{l}\text { Risque faible pour la } \\
\text { personne } \\
\text { Risque faible pour la } \\
\text { collectivité }\end{array}$ & $\begin{array}{l}\text { Acholeplasma spp. } \\
\text { Achromatium axoliferum } \\
\text { Acidaminobacter spp. }\end{array}$ \\
\hline RG2 & $\begin{array}{l}\text { Risque modéré pour la } \\
\text { personne } \\
\text { Risque faible pour la } \\
\text { collectivité }\end{array}$ & $\begin{array}{l}\text { Burkholderia multivorans } \\
\text { Escherichia coli } \\
\text { Salmonella enterica spp. }\end{array}$ \\
\hline RG3 & $\begin{array}{l}\text { Risque élevé pour la } \\
\text { personne } \\
\text { Risque faible pour la } \\
\text { collectivité }\end{array}$ & $\begin{array}{l}\text { Burkholderia pseudomallei } \\
\text { Brucella spp. } \\
\text { Francisella tularensis }\end{array}$ \\
\hline RG4 & $\begin{array}{l}\text { Risque élevé pour la } \\
\text { personne } \\
\text { Risque élevé pour la } \\
\text { collectivité }\end{array}$ & $\begin{array}{l}\text { Virus Alkhumra } \\
\text { Virus Ebola } \\
\text { Virus Nipah }\end{array}$ \\
\hline
\end{tabular}

Abréviation : spp., espèces

cliniques, contenant les spectres approuvés par Santé Canada, et les bases de données de recherche, qui contiennent un plus vaste ensemble de spectres, mais dont l'application clinique n'a pas été autorisée. II faut se procurer les extensions contenant les spectres des GR3 et des ABCSE séparément. Dans certains cas, les extensions sont soumises à des règlements d'importation imposants $(10,27,28)$, ce qui complique leur acquisition. Elles ne sont donc pas nécessairement aisément accessibles pour tous les laboratoires.

Grâce au système de surveillance de déclaration des incidents en laboratoire au Canada (DILC), I'Agence de la santé publique du Canada (ASPC) a la capacité d'évaluer le risque d'identification erronée par SM MALDI-TOF. Le système de surveillance de DILC a été mis en place en vertu de la Loi sur les agents pathogènes humains et les toxines, qui est entrée en vigueur en 2015 et qui rend obligatoire la déclaration des incidents de laboratoire à I'ASPC. Ce faisant, I'ASPC est en mesure de compiler des statistiques nationales sur la biosécurité et les problèmes liés à la biosécurité afin de dégager les tendances émergentes, pratiquement en temps réel.

La première fois que le système de surveillance de DILC a relevé des problèmes d'identification liés à la SM MALDI-TOF, cinq incidents d'exposition liés à l'utilisation de cette technologie avaient été signalés en neuf mois. Ces cinq incidents impliquaient des ABCSE du GR3 : Burkholderia pseudomallei, Francisella tularensis et des espèces de Brucella, qui sont responsables de la mélioïdose, de la tularémie et de la brucellose, respectivement. Ces microorganismes présentent un risque accru pour les techniciens de laboratoire, car ils sont facilement transmissibles par aérosol et leur croissance lente en milieu de culture standard fait en sorte qu'on ne soupçonne pas rapidement être en présence d'un ABCSE du GR3 (13,29-31). La mise en évidence de ces cinq incidents a déclenché une enquête 
visant à évaluer la nature et l'étendue du problème à l'échelle nationale.

Ce faisant, l'objectif de cette étude était de décrire les incidents d'exposition liés à l'utilisation de la SM MALDI-TOF au Canada de novembre 2015 à octobre 2017 et d'établir les facteurs de risque associés à ces incidents d'exposition.

\section{Méthodologie}

\section{Incidents admissibles}

Les incidents survenus du 6 novembre 2015 (date du premier incident) au 10 octobre 2017 (date des dernières données recueillies) ont été examinés. Les rapports ayant fait l'objet d'une évaluation plus approfondie ont été sélectionnés en fonction des critères $d$ 'inclusion suivants :

- Incidents avec ou sans exposition faisant directement référence à la SM MALDI-TOF; et

- Incidents avec ou sans exposition impliquant les espèces de Brucella, F. tularensis ou B. pseudomallei, dont les déclarations ne contenaient pas assez de renseignements permettant d'exclure avec certitude I'utilisation de la SM MALDI-TOF comme origine de l'incident.

Les incidents d'exposition étaient définis comme étant un « contact ou [une] proximité étroite avec des matières infectieuses ou des toxines pouvant respectivement causer une infection ou une intoxication » (6). Les incidents sans exposition étaient définis comme des cas de présence involontaire d'un microorganisme non autorisé en vertu du permis (p. ex. présence d'un microorganisme du GR3 dans une zone de confinement de niveau 2) (6).

Selon ces critères, 17 déclarations produites par 15 déclarants (organisations) ont été sélectionnées aux fins d'évaluation.

\section{Enquête}

Deux agents du programme DILC ont interrogé les déclarants en octobre et novembre 2017 en utilisant une liste de questions fournie à l'avance aux personnes interrogées. Le questionnaire visait à recueillir des données sur les points suivants : la possession d'un appareil de SM MALDI-TOF et d'extensions de bases de données; la méthodologie de l'analyse

(p. ex. a-t-elle été réalisée sur une table de travail à découvert?); les procédures opératoires normalisés (PONs) liés à l'utilisation de la SM MALDI-TOF; les facteurs déclencheurs pour I'adoption de PONs plus strictes; et l'opinion de la personne interrogée en ce qui concerne la cause de l'incident (est-il lié, en tout ou en partie, à l'identification erronée du microorganisme?). Le questionnaire portait également sur la présence de renseignements sur le spécimen ( $p$. ex. diagnostic soupçonné, antécédents médicaux et antécédents de voyage du patient) que le personnel médical ayant fait la demande d'identification aurait pu fournir et qui auraient pu alerter les employés de laboratoire sur le risque. L'admissibilité d'un incident à la présente étude était confirmée une fois que le déclarant responsable corroborait que l'incident résultait (au moins en partie) de l'identification erronée ou de la non-identification du microorganisme par un appareil de SM MALDI-TOF.

\section{Analyse}

Les réponses au questionnaire ont été consignées dans une feuille de calcul mise au point spécialement pour cette enquête. La version 2010 du logiciel Excel a été utilisée pour réaliser une analyse statistique descriptive standard visant à évaluer et à dégager les caractéristiques communes.

\section{Résultats}

Parmi les 15 organisations jointes par l'équipe de l'étude pour obtenir des renseignements sur les 17 incidents admissibles, 12 possédaient un appareil de SM MALDI-TOF. Deux des incidents survenus dans les trois organisations n'ayant pas I'appareil étaient tout de même partiellement liés à I'utilisation de la SM MALDI-TOF. En effet, les déclarants ont indiqué que ces spécimens avaient fait l'objet d'une identification erronée ou d'une non-identification par SM MALDI-TOF dans un autre laboratoire avant d'arriver dans leur organisation aux fins de confirmation.

Les déclarants des 12 organisations possédant un appareil de SM MALDI-TOF ont confirmé que six incidents étaient partiellement ou entièrement attribuables à la confiance accordée aux résultats d'identification par SM MALDI-TOF. En ajoutant les deux cas mentionnés ci-dessus (analyses de spécimens effectuées dans un autre laboratoire), on compte huit incidents liés à l'identification erronée ou à la non-identification du spécimen par SM MALDI-TOF (voir la figure 1). Ces incidents représentent plus du tiers $(n=8 / 17)$ des incidents liés aux trois agents biologiques retenus qui ont été signalés au système de surveillance DILC du 6 novembre 2015 au 10 octobre 2017.

Selon l'information fournie dans les huit déclarations d'incident (dont deux incidents sans exposition), 39 techniciens de laboratoire ont été exposés. Burkholderia pseudomallei était l'agent biologique en cause dans trois de ces incidents; les espèces de Brucella étaient impliquées dans trois autres incidents et $F$. tularensis était en cause dans deux incidents (voir le tableau 2).

Dans cinq des huit incidents, les déclarants ont indiqué que I'appareil de SM MALDI-TOF de leur organisation comprenait les bases de données de référence cliniques et de recherche. Dans un incident, l'appareil ne comportait que la base de données cliniques; dans deux incidents, le déclarant ne savait pas quel type de base de données de référence était utilisée. Toutefois, dans cinq des incidents où les deux types de bases de données étaient accessibles, toutes les analyses ont été réalisées en consultant uniquement la base de données cliniques.

Dans les huit cas liés à I'utilisation de la SM MALDI-TOF, I'incident est survenu à l'étape de préparation du spécimen et les analyses ont été réalisées sur table de travail à découvert, directement sur le spécimen. Par ailleurs, pour chaque incident survenu dans une organisation équipée d'un appareil de SM MALDI-TOF $(n=6)$, le déclarant a mis au point, à la suite de l'incident, des PONs conçues spécialement pour l'utilisation de 


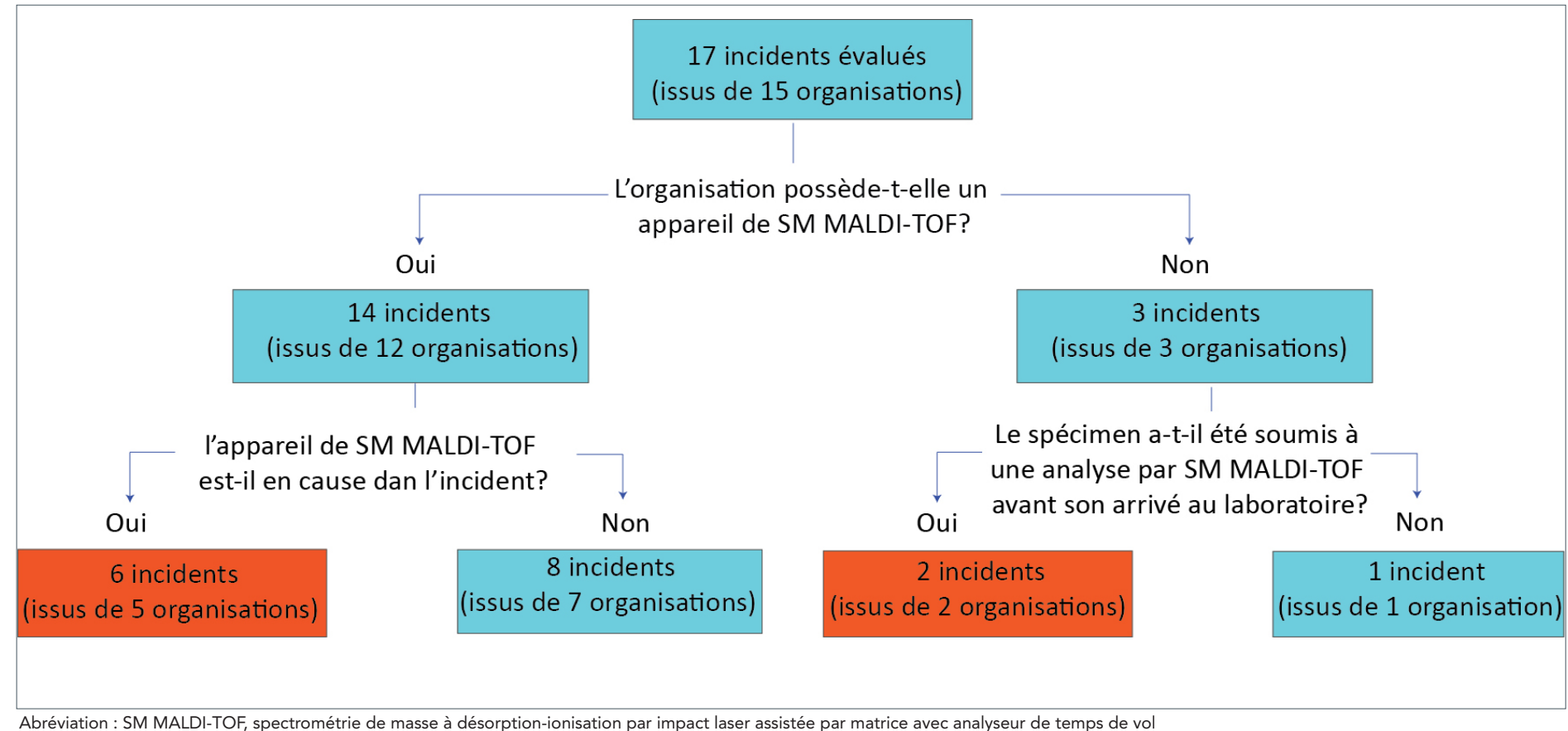

Abréviation : SM MALDI-TOF, spectrométrie de masse à désorption-ionisation par impact laser assistée par matrice avec analyseur de temps de vol

cette technologie. Les éléments qui ont incité l'instauration de ces PONs comprennent la croissance lente des coccobacilles à Gram négatif $(n=2)$, l'absence de résultats d'identification $(n=2)$, la région géographique d'où provient le spécimen $(n=1)$ et le type d'échantillon $(n=1)$.

Tableau 2 : Agents biologiques impliqués et nombre de personnes exposées par incident

\begin{tabular}{|c|l|c|}
\hline $\begin{array}{c}\text { Numéro } \\
\text { d'incident }\end{array}$ & Agent biologique impliqué & $\begin{array}{c}\text { Nombre de } \\
\text { personnes } \\
\text { exposées }\end{array}$ \\
\hline 1 & Burkholderia pseudomallei & 1 \\
\hline 2 & B. pseudomallei & 1 \\
\hline 3 & Francisella tularensis & 13 \\
\hline 4 & F. tularensis & 4 \\
\hline 5 & Brucella abortus & 15 \\
\hline 6 & B. pseudomallei & 5 \\
\hline 7 & Brucella melitensis & $\mathrm{n} / \mathrm{a}$ \\
\hline 8 & Brucella spp & $\mathrm{n} / \mathrm{a}$ \\
\hline
\end{tabular}

Finalement, dans les huit incidents liés à l'utilisation de la SM MALDI-TOF, les spécimens sont arrivés sans être accompagnés des renseignements cliniques ou des antécédents de voyage du patient (c.-à-d. des informations qui permettraient de soupçonner la présence d'ABCSE du GR3).

\section{Discussion}

Il s'agit de la première étude menée à l'échelle nationale ayant évalué les incidents en laboratoire liés à l'identification erronée ou à la non-identification des ABCSE du GR3 par les appareils de
SM MALDI-TOF. Au total, 39 personnes ont été exposées à des ABCSE du GR3 dans six incidents d'exposition liés à l'utilisation de la SM MALDI-TOF. Ces microorganismes correspondent à ceux ayant été impliqués dans d'autres incidents signalés dans la littérature $(3,10-12,15,19,22)$.

Un facteur de risque associé aux incidents d'exposition était la confiance accordée au logiciel de base de données cliniques de référence. Selon les déclarants, bien que les bases de données cliniques et les bases de données de recherche de référence aient été disponibles dans la plupart des cas, seules les bases de données cliniques avaient été utilisées dans les cas où les incidents d'exposition sont survenus. En effet, les deux types de base de données ne peuvent être utilisés simultanément, et le passage d'une base de données à l'autre nécessite de longues étapes qui ne conviennent pas aux charges de travail actuelles. Par conséquent, si les bases de données de référence complémentaires (comme les bases de données de recherche ou les bases de données de sécurité) peuvent contribuer à atténuer le risque d'identification erronée en fournissant davantage de spectres de comparaison, leur utilité reste limitée étant donné qu'il est impossible de les combiner activement aux autres types de bases de données. Ces résultats correspondent à ceux d'autres rapports qui ont été publiés et qui laissent croire que les bases de données cliniques de SM MALDI-TOF ne permettent pas d'identifier avec certitude certains ABCSE du GR3 $(9,11,12,20,22)$.

Un deuxième facteur de risque associé aux incidents d'exposition est la préparation des spécimens sur table de travail à découvert. En effet, tous les incidents sont survenus pendant la préparation des spécimens et toutes les analyses ont été réalisées directement sur le spécimen, sur table de travail à découvert. Cependant, cette approche ne reflète pas les recommandations du fabricant, qui suggèrent plutôt d'effectuer les analyses après une extraction (32) qui, dans la plupart des cas, désactive 
efficacement les agents pathogènes et atténue donc le risque d'exposition.

Un troisième facteur de risque associé aux incidents d'exposition est lié au manque de renseignements d'alerte dans les demandes d'analyse de laboratoire. Les trois maladies provoquées par les microorganismes en cause sont difficiles à identifier, car elles sont associées à des symptômes non spécifiques qui sont facilement attribués à d'autres maladies plus fréquentes (8,25-27). Par conséquent, il se peut que les cliniciens ne soupçonnent pas un diagnostic d'infection par un ABCSE du GR3 et que leurs formulaires de demande ne fournissent pas les renseignements d'alerte (c.-à-d. diagnostic soupçonné, antécédents de voyage du patient) qui pourraient entraîner l'adoption de mesures de sécurité adéquates pour la manipulation des spécimens des patients en laboratoire $(30,33)$.

La présente étude a mis en évidence deux incidents liés à des spécimens ayant été mal identifiés par un appareil de SM MALDI-TOF dans un autre laboratoire. Ces incidents indiquent que des expositions reliées à un seul spécimen peuvent survenir dans plusieurs laboratoires.

\section{Points forts et limites}

Le principal point fort de cette étude vient de la déclaration obligatoire, normalisée et détaillée des incidents en laboratoire. Ces déclarations obligatoires créent un point de départ pour évaluer l'évolution du problème dans le temps.

Il y a cependant quelques limites dont il faut tenir compte. L'échantillon d'incidents détectés est probablement incomplet, et ce, pour deux raisons. Premièrement, les critères d'inclusion peuvent avoir fait en sorte que certains incidents aient été omis, car il n'y a présentement aucun moyen efficace de relever avec certitude tous les incidents pouvaient être liés à l'utilisation d'appareils de SM MALDI-TOF parmi tous les incidents de laboratoires signalés au système de surveillance de DILC. Deuxièmement, cette enquête ne portait que sur les incidents impliquant les trois microorganismes sélectionnés. En effet, des cas d'identification erronée ou de non-identification par les appareils de SM MALDI-TOF ont aussi été signalés pour certains microorganismes du GR2 (34). Enfin, l'échantillon d'incidents d'expositions analysés est fondamentalement instable, compte tenu du petit nombre d'incidents survenus sur une période de deux ans. D'autres études montrant les tendances au fil du temps permettront d'avoir une meilleure idée de l'étendue du problème.

\section{Conclusion}

Cette étude nationale de surveillance des incidents de laboratoire liés à l'identification erronée des ABCSE du GR3 par les appareils de SM MALDI-TOF a mise en évidence trois facteurs de risque : la confiance accordée aux bases de données cliniques de référence; la préparation des spécimens en table de travail à découvert; et l'absence de renseignements d'alerte dans les demandes d'analyse de laboratoire. Cette information peut servir à faire connaître les limites de la technologie de la SM MALDI-TOF et à encourager la mise au point de mesures permettant d'éviter de tels incidents. Elle constitue également un point de départ pour la surveillance dans le temps. Le Centre de la biosûreté poursuit l'enquête et continue de travailler avec les parties prenantes pour s'attaquer au problème et améliorer les mesures de biosécurité.

\section{Déclaration des auteurs}

D. P. N. - Recherche, enquête, analyse des données, rédaction - Ébauche initiale et rédaction - Révision et édition M. S. - Enquête, rédaction - Révision, édition, supervision M. H. - Rédaction - Édition, supervision

\section{Conflit d'intérêt}

Aucun.

\section{Remerciements}

Nous tenons à remercier tous les déclarants avec qui nous avons communiqué au cours du processus d'enquête pour leur collaboration active. Nous tenons également à remercier tous les titulaires de permis et les agents de la sécurité biologique de partout au Canada pour la soumission de rapports de haute qualité.

\section{Financement}

Ce travail a été appuyé par l'Agence de la santé publique du Canada dans le cadre de son mandat de base.

\section{Références}

1. Calderaro A, Arcangeletti MC, Rodighiero I, Buttrini M, Gorrini C, Motta F, Germini D, Medici MC, Chezzi C, De Conto F. Matrix-assisted laser desorption/ionization time-offlight (MALDI-TOF) mass spectrometry applied to virus identification. Sci Rep 2014 Oct;4(6803):6803. https://dx.doi. org/10.1038\%2Fsrep06803. PubMed (https://www.ncbi.nlm. nih.gov/pmc/articles/PMC4213803/)

2. Ministère de la Santé et des Services Sociaux. Projet de réorganisation des laboratoires.Info Labo Bulletin. 2017 June;6(2). http://www.cisss-ca.gouv.qc.ca/fileadmin/ documents/Professionnels/Laboratoires/Info-Labo-2 Juin_2017.pdf

3. Singhal N, Kumar M, Kanaujia PK, Virdi JS. MALDI-TOF mass spectrometry: an emerging technology for microbial identification and diagnosis. Front Microbiol 2015 Aug;6(791):1-16. PubMed (https://www.ncbi.nlm.nih.gov/ pubmed/26300860?dopt=Abstract)

4. Clark AE, Kaleta EJ, Arora A, Wolk DM. Matrix-assisted laser desorption ionization-time of flight mass spectrometry: a fundamental shift in the routine practice of clinical microbiology. Clin Microbiol Rev 2013 Jul;26(3):547-603. http://dx.doi.org/10.1128/CMR.00072-12. PubMed (https:// www.ncbi.nlm.nih.gov/pubmed/23824373?dopt=Abstract)

5. Saraswathy N, Ramalingam P. Protein Identification by Peptide Mass Fingerprinting. In: Saraswathy N, Ramalingam P, editors. 
Concepts and Techniques in Genomics and Proteomics. 1th ed. Cambridge: Woodhead Publishing; 2011. p. 185-92.

6. Agence de la santé publique du Canada. Norme canadienne sur la biosécurité (NCB), Deuxième édition. Ottawa: Agence de la santé publique du Canada; 2015. https://www.canada. $\mathrm{ca} / \mathrm{fr} /$ sante-publique/services/normes-lignes-directricescanadiennes-biosecurite/deuxieme-edition.html

7. Bizzini A, Greub G. Matrix-assisted laser desorption ionization time-of-flight mass spectrometry, a revolution in clinical microbial identification. Clin Microbiol Infect 2010 Nov;16(11):1614-9. http://dx.doi.org/10.1111/j.14690691.2010.03311.x. PubMed (https://www.ncbi.nlm.nih.gov/ pubmed/20636422?dopt=Abstract)

8. L'Agence canadienne des médicaments et des technologies de la santé. Exactitude et efficacité clinique de la spectrométrie de masse MALDI-TOF dans l'identification d'agents pathogènes. Rapports d'examen rapide. Oct 2015:165. https://www.cadth.ca/fr/exactitude-et-efficacite-cliniquede-la-spectrometrie-de-masse-maldi-tof-dans-lidentification

9. Cunningham SA, Patel R. Importance of using Bruker's security-relevant library for Biotyper identification of Burkholderia pseudomallei, Brucella species, and Francisella tularensis. J Clin Microbiol 2013 May;51(5):1639-40. http:// dx.doi.org/10.1128/JCM.00267-13. PubMed (https://www. ncbi.nlm.nih.gov/pubmed/23447635?dopt=Abstract)

10. Dingle TC, Butler-Wu SM, Abbott AN. Accidental exposure to Burkholderia pseudomallei in the laboratory in the era of matrix-assisted laser desorption ionization-time of flight mass spectrometry. J Clin Microbiol 2014 Sep;52(9):3490-1. http:// dx.doi.org/10.1128/JCM.01238-14. PubMed (https://www. ncbi.nlm.nih.gov/pmc/articles/PMC4313193/)

11. Jang HR, Lee CW, Ok SJ, Kim MJ, Bae MJ, Song S, Yi J, $\mathrm{Kim} \mathrm{KH}$. Melioidosis presenting as a mycotic aneurysm in a Korean patient, diagnosed by $16 \mathrm{~S}$ rRNA sequencing and matrix-assisted laser desorption/ionization time-of-flight mass spectrometry. Int J Infect Dis 2015 Sep;38:62-4. http://dx.doi. org/10.1016/j.ijid.2015.07.012. PubMed (https://www.ncbi. nlm.nih.gov/pubmed/26216763?dopt=Abstract)

12. Keller PM, Bruderer $V$, Müller F. Restricted Identification of Clinical Pathogens Categorized as Biothreats by MALDI-TOF Mass Spectrometry. J Clin Microbiol 2016;54(3):816. http:// dx.doi.org/10.1128/JCM.03250-15. PubMed (https://www. ncbi.nlm.nih.gov/pubmed/26763958?dopt=Abstract)

13. Kolecka A, Sanguinetti M, Boekhout T, Posteraro B. MALDI-TOF in Microbiology. First ed. Kostrzewa M and Schubert S, editors. Bremen: Caister Academic Press; 2016.

14. Lévesque S, Dufresne PJ, Soualhine H, Domingo MC, Bekal S, Lefebvre B, Tremblay C. A Side by Side Comparison of Bruker Biotyper and VITEK MS: Utility of MALDI-TOF MS Technology for Microorganism Identification in a Public Health Reference Laboratory. PLoS One 2015 Dec;10(12):e0144878. http:// dx.doi.org/10.1371/journal.pone.0144878. PubMed (https:// www.ncbi.nlm.nih.gov/pubmed/26658918?dopt=Abstract)

15. Lista F, Reubsaet FA, De Santis R, Parchen RR, de Jong $A L$, Kieboom J, van der Laaken AL, Voskamp-Visser IA, Fillo S, Jansen HJ, Van der Plas J, Paauw A. Reliable identification at the species level of Brucella isolates with MALDI-TOFMS. BMC Microbiol 2011 Dec;11:267. http://dx.doi. org/10.1186/1471-2180-11-267. PubMed (https://www.ncbi. nlm.nih.gov/pubmed/22192890?dopt=Abstract)

16. Mitchell PK, Campbell C, Montgomery MP, Paoline J, Wilbur C, Posivak-Khouly L, Garafalo K, Elrod M, Liu L, Weltman A. Notes from the Field: Travel-Associated Melioidosis and Resulting Laboratory Exposures - United States, 2016. MMWR Morb Mortal Wkly Rep 2017 Sep;66(37):1001-2. http://dx.doi. org/10.15585/mmwr.mm6637a8. PubMed (https://www.ncbi. nlm.nih.gov/pubmed/28934180?dopt=Abstract)

17. Saffert RT, Cunningham SA, Ihde SM, Jobe KE, Mandrekar J, Patel R. Comparison of Bruker Biotyper matrix-assisted laser desorption ionization-time of flight mass spectrometer to BD Phoenix automated microbiology system for identification of gram-negative bacilli. J Clin Microbiol 2011 Mar;49(3):887-92. http://dx.doi.org/10.1128/JCM.01890-10. PubMed (https:// www.ncbi.nlm.nih.gov/pubmed/21209160?dopt=Abstract)

18. Santos CR, Francisco E, Mazza M, Carolina A, Padovan B. Impact of MALDI-TOF MS in Clinical Mycology: Progress and Barriers in Diagnostics. In: Shah HN, Gharbia SE, editors. MALDI-TOF and Tandem MS for Clinical Microbiology. First ed. London: John Wiley and Sons Ltd; 2017. p. 211-30.

19. Suttisunhakul $V$, Pumpuang $A$, Ekchariyawat $P$, Wuthiekanun V, Elrod MG, Turner P, Currie BJ, Phetsouvanh R, Dance DA, Limmathurotsakul D, Peacock SJ, Chantratita N.'Matrix-assisted laser desorption/ionization time-of-flight mass spectrometry for the identification of Burkholderia pseudomallei from Asia and Australia and differentiation between Burkholderia species. PLoS One 2017 Apr;12(4):e0175294. http://dx.doi. org/10.1371/journal.pone.0175294. PubMed (https://www. ncbi.nlm.nih.gov/pubmed/28384252?dopt=Abstract)

20. Tracz DM, Antonation KS, Corbett CR. Verification of a Matrix-Assisted Laser Desorption lonization-Time of Flight Mass Spectrometry Method for Diagnostic Identification of High-Consequence Bacterial Pathogens. J Clin Microbiol 2016 Mar;54(3):764-7. http://dx.doi.org/10.1128/

JCM.02709-15. PubMed (https://www.ncbi.nlm.nih.gov/ pubmed/26677252?dopt=Abstract)

21. Tracz DM, Tyler AD, Cunningham I, Antonation KS, Corbett CR. Custom database development and biomarker discovery methods for MALDI-TOF mass spectrometry-based identification of high-consequence bacterial pathogens. J Microbiol Methods 2017 Mar;134:54-7. http://dx.doi. org/10.1016/j.mimet.2017.01.009. PubMed (https://www.ncbi. $\mathrm{nlm}$.nih.gov/pubmed/28108323?dopt=Abstract)

22. Wang $H$, Chen $Y L$, Teng $S H, X u Z P, X u$ YC, Hsueh PR. Evaluation of the Bruker Biotyper Matrix-Assisted Laser Desorption/lonization Time-of-Flight Mass Spectrometry System for Identification of Clinical and Environmental Isolates of Burkholderia pseudomallei. Front Microbiol 2016 Apr;7(415):1-8. https://doi.org/10.3389/

fmicb.2016.00415. PubMed (https://www.ncbi.nlm.nih.gov/ pubmed/27092108?dopt=Abstract)

23. Ferreira L, Vega Castaño $S$, Sánchez-Juanes $F$, González-Cabrero S, Menegotto F, Orduña-Domingo A, González-Buitrago JM, Muñoz-Bellido JL. Identification of Brucella by MALDI-TOF Mass Spectrometry. Fast and Reliable Identification from Agar Plates and Blood Cultures. PLoS ONE. 2010 Dec;5(12):-e14235. https://dx.doi. org/10.1371\%2Fjournal.pone.0014235. PubMed (https://www. ncbi.nlm.nih.gov/pmc/articles/PMC2997794/)

24. Karger A, Melzer F, Timke M, Bettin B, Kostrzewa M, Nöckler K, Hohmann A, Tomaso H, Neubauer H, Al Dahouk S. Interlaboratory comparison of intact-cell matrix assisted laser desorption ionization - time of flight mass spectrometry results matrix-assisted laser desorption ionization mass spectrometry results for identification and differentiation of Brucella spp. J Clin Microbiol 2013;51(9):3123-6. http://dx.doi.org/10.1128/ JCM.01720-13. PubMed (https://www.ncbi.nlm.nih.gov/ pubmed/23850950?dopt=Abstract)

25. Lau SK, Sridhar S, Ho CC, Chow WN, Lee KC, Lam CW, Yuen KY, Woo PC. Laboratory diagnosis of melioidosis: past, present and future. Exp Biol Med 2. 2015 Jun;240(6):742-51. https:// doi.org/10.1177/1535370215583801. PubMed (https://www. ncbi.nlm.nih.gov/pubmed/25908634) 
26. Walewski $\bigvee$, Méchaï $F$, Billard-Pomares $T$, Juguet $W$, Jauréguy $F$, Picard B, Tandjaoui-Lambiotte Y, Carbonnelle E, Bouchaud O. MALDI-TOF MS contribution to diagnosis of melioidosis in a nonendemic country in three French travellers. New Microbes New Infect 2016 Apr;12:31-4. http://dx.doi.org/10.1016/j. nmni.2016.04.004. PubMed (https://www.ncbi.nlm.nih.gov/ pubmed/27222715?dopt=Abstract)

27. Dual-use export controls. Brussels: European Commission; 2018 [Consulté 2018 Jan 20]. http://ec.europa.eu/trade/ import-and-export-rules/export-from-eu/dual-use-controls/

28. COUNCIL REGULATION (EC) Setting up a Community regime for the control of exports, transfer, brokering and transit of dual-use items, No 428/2009. http://eur-lex.europa.eu/legalcontent/EN/TXT/?gid=1489490204085\&uri=CELEX:0200 9R0428-20161116 (May 5, 2009)

29. Yagupsky P, Baron EJ. Laboratory exposures to brucellae and implications for bioterrorism. Emerg Infect Dis 2005 Aug;11(8):1180-5. http://dx.doi.org/10.3201/ eid1108.041197. PubMed (https://www.ncbi.nlm.nih.gov/ pubmed/16102304?dopt=Abstract)

30. Pedati C, House J, Hancock-Allen J, Colton L, Bryan K, Ortbahn D, Kightlinger L, Kugeler K, Petersen J, Mead P, Safranek T, Buss B. Notes from the Field: Increase in Human Cases of Tularemia--Colorado, Nebraska, South Dakota, and Wyoming, January-September 2015. MMWR Morb Mortal Wkly Rep 2015 Dec;64(47):1317-8. http://dx.doi. org/10.15585/mmwr.mm6447a4. PubMed (https://www.ncbi. nlm.nih.gov/pubmed/26632662?dopt=Abstract)

31. Wagar E. Bioterrorism and the Role of the Clinical Microbiology Laboratory. Clin Microbiol Rev 2016 Jan;29(1):175-89. http://dx.doi.org/10.1128/

CMR.00033-15. PubMed (https://www.ncbi.nlm.nih.gov/ pubmed/26656673?dopt=Abstract)

32. Bruker Daltonics. Instructions for Use MALDI Biotarget 48. Bruker Daltonics; 2011 December. https://www.criver.com/ sites/default/files/resources/InstructionsforUsingMALDIBiotarg et48.pdf

33. Traxler RM, Lehman MW, Bosserman EA, Guerra MA, Smith TL. A literature review of laboratory-acquired brucellosis. J Clin Microbiol 2013 Sep;51(9):3055-62. http://dx.doi.org/10.1128/ JCM.00135-13. PubMed (https://www.ncbi.nlm.nih.gov/ pubmed/23824774?dopt=Abstract)

34. Bilecen K, Yaman G, Ciftci U, Laleli YR. Performances and Reliability of Bruker Microflex LT and VITEK MS MALDI-TOF Mass Spectrometry Systems for the Identification of Clinical Microorganisms. BioMed Res Int 2015;2015(516410):1-18. http://dx.doi.org/10.1155/2015/516410. PubMed (https:// www.ncbi.nlm.nih.gov/pubmed/26793718?dopt=Abstract)

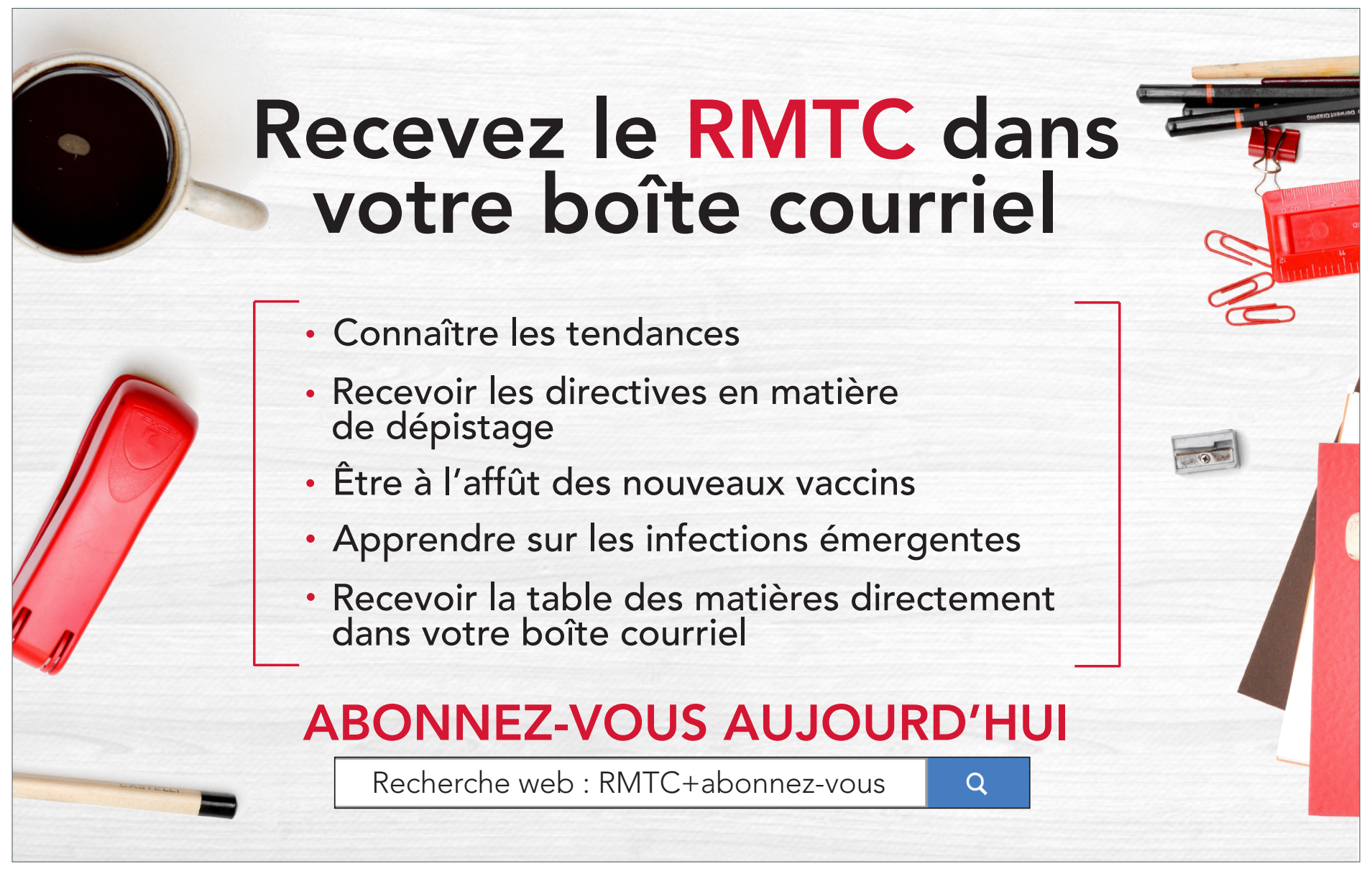

